\title{
Evaluation of Channel Assignment Algorithms in a Dense Real World WLAN
}

\author{
A. Raschellà, M. Mackay, F. Bouhafs \\ Department of Computer Science \\ Liverpool John Moores University \\ Liverpool, United Kingdom \\ \{a.raschella, m.i.mackay, f.bouhafs\}@1jmu.ac.uk
}

\author{
B. Ivar Teigen \\ Domos AS \\ Oslo, Norway \\ bjorn@domos.no
}

\begin{abstract}
This paper addresses the problem of Access Point (AP) channel assignment in dense IEEE 802.11 Wireless Local Area Networks (WLANs) implemented in a real world scenario, based on a housing complex located in Oslo, Norway. Currently, the APs composing this housing complex are centrally configured through a channel selection approach based on a genetic algorithm that aims to minimize the cumulative interference experienced by each AP. In this work, we present the performance of an alternative channel assignment algorithm in comparison to the existing genetic one. More specifically, the algorithm investigated in this work aims to minimize a networkwide parameter called interference impact, which represents the interference caused by an AP to all the other APs in the neighbourhood. Moreover, the algorithm is implemented using the spectrum programming architecture Wi-5 based on Software-Defined Networking (SDN). Although the benefits of this algorithm have been demonstrated in simulated environments, this work presents its first evaluation in a dense real world scenario. The performance analysis illustrates the important gains obtained in terms of the data transmissions quality through the proposed algorithm compared against the AP channel selection approach currently implemented in the considered scenario.
\end{abstract}

Keywords-Wi-Fi, Dense Wireless Local Area Networks (WLANs), AP channel assignment, Interference mitigation, Software-Defined Networking, Wi-5

\section{INTRODUCTION}

Wi-Fi technology is now ubiquitous and has made its way into many densely populated areas such as housing complexes, airports, train stations, shopping malls and university campuses. These dense areas are covered by IEEE 802.11 Wireless Local Area Networks (WLANs) relying on Radio Frequency (RF) overlapping Access Points (APs), which guarantee good signal coverage and redundant connectivity to the users. However, the fact that these APs are distributed without proper coordination and work on unlicensed Wi-Fi frequency bands, has resulted in rising levels of unwanted interference and congestion, affecting the overall performance of the network [1]. As such, there is a rich body of Wi-Fi management solutions available in the literature aiming to optimize the use of the Wi-Fi frequency bands through radio resource management and guarantee continuous connection to their clients in congested networks. Radio management solutions in $\mathrm{Wi}-\mathrm{Fi}$ networks include, for instance, AP channel assignment optimization [1]-[9] and AP user allocation [10]-[12].

Undoubtedly, an efficient AP channel assignment plays a major role in mitigating interference and, therefore, improving the performance of dense IEEE 802.11 WLANs. In this paper we address the problem of AP channel assignment in a dense
WLAN implemented in a housing complex located in Oslo, Norway. In the scenario, Domos ${ }^{1}$ has developed a cloud-based solution that uses a channel selection approach for configuring the APs composing the housing complex based on a genetic algorithm. This algorithm tries to optimize a network cost function that depends on the power detected in each AP from all other APs of the network. Therefore, Domos relies on centralised coordination aiming to minimize the cumulative interference experienced by the APs of the housing complex. The solutions presented in [2]-[6] also propose algorithms that aim to optimize the cumulative interference in a similar way to how Domos algorithm does. The main shortcoming of this approach is that it does not give the necessary information that would help identify the optimal configuration for the whole network [1].

In contrast, our solutions presented in [1], and [7]-[9] have introduced a novel algorithm able to minimise the so-called interference impact, which represents the network-wide impact of each AP towards all the other APs in the considered Wi-Fi network. In [7] we have proposed the first version of this algorithm, which was then extended in [1] and [8] by the inclusion of a transmit power control taking into consideration the users' Quality of Service (QoS) requirements. In these works we have demonstrated how our approach provides better performance in terms of interference, Signal-toInterference-plus-Noise Ratio (SINR), spectral efficiency and users' satisfaction compared to the state of the art. Moreover, in [9] we have evaluated the channel assignment algorithm in an apartment block by means of OPNET simulations of Wi-Fi in the $2.4 \mathrm{GHz}$ band showing encouraging results in terms of throughput. In these previous contributions, we proposed the implementation of our algorithm in a simulated framework built on top of the Wi-5 architecture [13] based on SoftwareDefined Networking (SDN) and developed in the context of the Horizon 2020 (H2020) funded Wi-5 (What to do With the Wi-Fi Wild West) project [14]. The project has developed an architecture for radio resource management and optimisation suitable for coordinating dense environments such as airports and housing complexes. In this architecture, the $\mathrm{Wi}-5$ controller is the central entity that manages the Wi-Fi networks and executes the AP channel assignment algorithm.

In this paper, we demonstrate for the first time the benefits of our AP channel selection algorithm in a dense real world scenario represented by the Domos housing complex. Specifically, we illustrate how the optimization of the interference impact allows us to improve the performance of an algorithm that optimizes the cumulative interference instead. Note that the main differences between the interference impact and the cumulative interference will be clarified in Section IV.

\footnotetext{
${ }^{1}$ http://www.domos.no/
} 
The rest of the paper is organized as follows: in Section II we describe the scenario and the system model considered in this paper for the assessment of the Wi-5 AP channel assignment algorithm against the Domos channel assignment one. Then, in Section III we provide a detailed explanation of both algorithms. In Section IV we illustrate the performance obtained by both algorithms and the improvements achieved through the Wi-5 approach. Finally, conclusions and future works are presented in Section V.

\section{SCENARIO AND SYSTEM MODEL}

The scenario considered in this paper is a housing complex located in Oslo, Norway, consisting of 119 802.11n APs, 40 of which are controlled by Domos and can be configured to work on specific channels. The set of 119 APs is represented by the graph shown in Fig. 1 where each AP is a node with edges connecting to each Wi-Fi AP that can be detected through a scan. The graph illustrated in the figure has been drawn using the kamada-kawai method for graph visualisation as implemented in the NetworkX Python package. Moreover, the graph was constructed using scans performed by the APs controlled by Domos.

Specifically, each scan performed by each Domos AP dwells for $200 \mathrm{~ms}$ on each channel, and for each detected AP it reports: 1) the Basic Service Set Identifier (BSSID), 2) the Service Set Identifier (SSID), 3) the channel, 4) the channel width, and 5) the Received Signal Strength Indicator (RSSI). The edges in the graph are directed from the detected AP to the scanning AP controlled by Domos. Therefore, in the figure, bidirectional links connect Domos APs. Data on the RSSI of the scanned APs are stored in the edges of the graph.

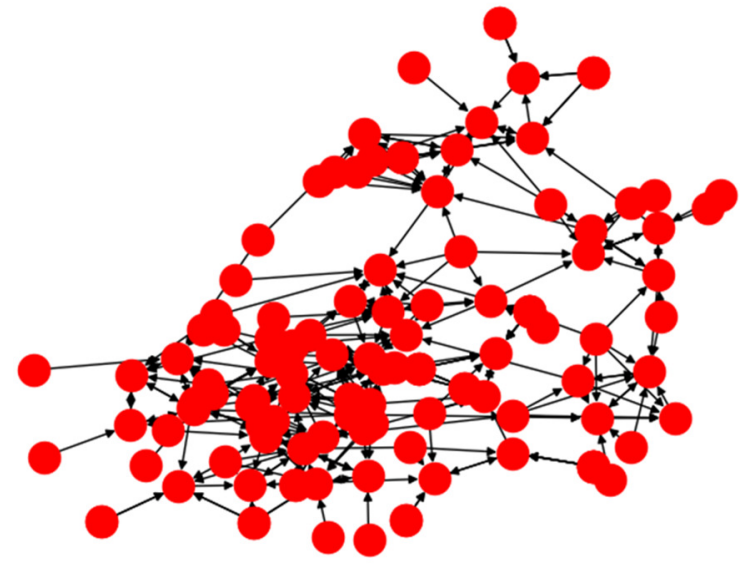

Fig. 1. Considered Scenario

Moreover, the collected data represent also the input of Domos algorithm, which is implemented in a cloud-based centralized network management platform, and Wi-5 algorithm that is implemented in a platform based on the Wi5 architecture [13]. Based on the collected data, both algorithms provide the channel configuration for the managed APs included in the considered scenario. Fig. 2 illustrates the system model that implements the AP channel assignment problem in the housing complex considered in this paper.

The centralized network management platform based on the Wi-5 architecture and that implements the Wi-5 algorithm is illustrated in Fig. 3. Specifically, the Wi-5 controller offers flexibility and cross-layer management, as it is able to obtain monitoring information about the status of the network and execute relevant algorithms in a Decision-Making module. In this platform, the input of the algorithm can be updated in real-time and are dependent on the actual characteristics of the employed RF channels as well as the arrangement of the network.

For the specific study presented in this paper, the abovementioned collected data have been injected in the Decision-Making module that provides the APs channel configuration. Further details on the use of the data collected in the algorithms together with their analytical details will be provided in the next section.

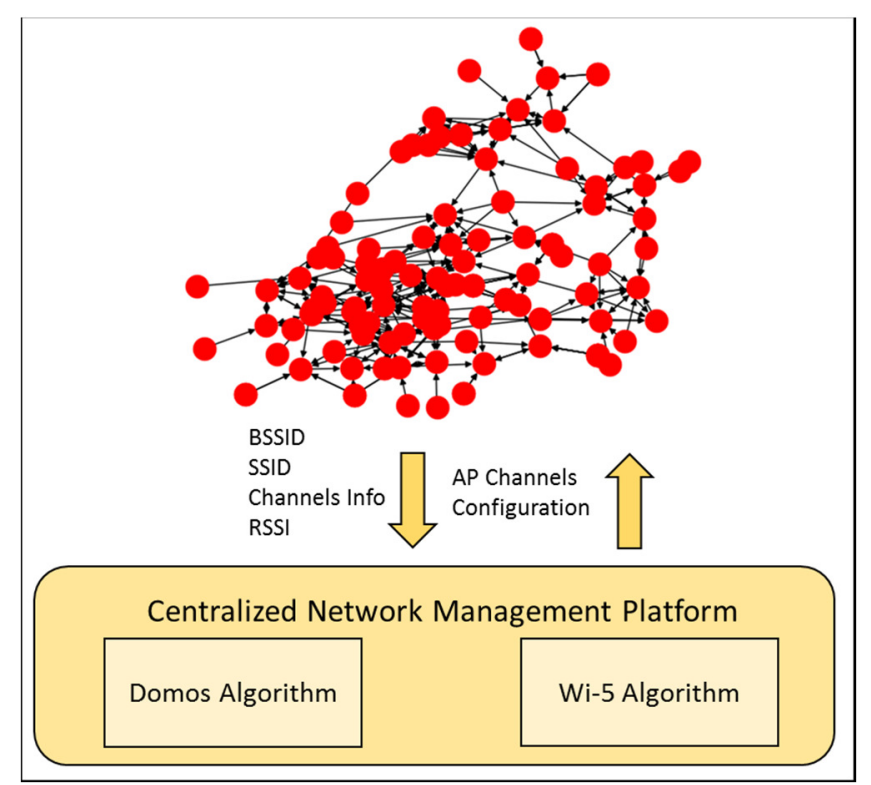

Fig. 2. Centralized Network Management Platform

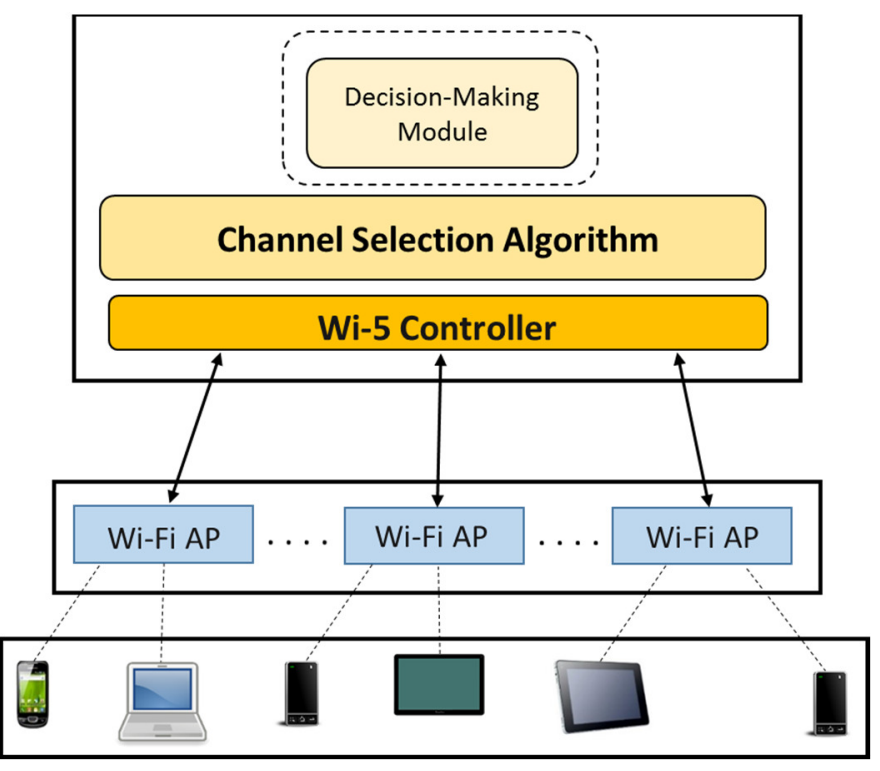

Fig. 3. Platform based on the Wi-5 Architecture [13]

\section{AP CHANNEL ASSIGNMENT ALGORITHMS}

\section{A. Domos Algorithm}

This section presents the algorithm implemented in the Domos cloud-based centralized network management platform. Specifically, Domos solution utilizes a channel assignment approach based on a genetic algorithm [15] in order to optimize a cost function, which we will explain throughout this section. Each AP is located in a position within 
a vector with length $N$, where $N$ is the total number of APs. Each possible channel assignment for the $N$ APs is called individual. The genetic algorithm is initialized by creating a population $P$ consisting of 1000 random individuals. The channels are randomly selected from a set that includes the Industrial, Scientific and Medical (ISM) channels 1, 6, and 11 (i.e., channels centred at $2.412 \mathrm{GHz}, 2.437 \mathrm{GHz}$ and 2.462 $\mathrm{GHz}$, respectively). Each individual $i$ in the population $P$ is evaluated by the cost function $C_{i}$ that assigns a cost to it.

Let us now define the cost function $C_{i}$ for each individual $i$ as the sum of edges in the graph where the channels at the joined nodes are less than $20 \mathrm{MHz}$ apart and included in a set $E$, with each edge $e$ weighted by a function $w_{e}$ that depends on the scanned RSSI at edge $e$, i.e., $R S S I_{e}$. This means that the cost of an edge between two APs configured to work on channels that are more than $20 \mathrm{MHz}$ apart is always zero. Specifically, $w_{e}$ is defined as follows:

$$
w_{e}=\max \left(0, \min \left(R S S I_{e} / a+b, 1\right)\right)
$$

Where $a$ and $b$ are shaping parameters to be defined. The values of these parameters considered in the Domos facilities have been chosen empirically in order to match RSSI values with relevant observed levels of interference, resulting then in a weight of 1 for RSSI values between $-65 \mathrm{dBm}$ and $0 \mathrm{dBm}$, while the weights are linearly increased for RSSI values included between $-90 \mathrm{dBm}$ and $-65 \mathrm{dBm}$. Therefore, for the scenario analysed in this paper $a=25$ and $b=3.6$ have been considered in (1). Fig. 4 illustrates how the weight of an edge varies as a function of the RSSI.

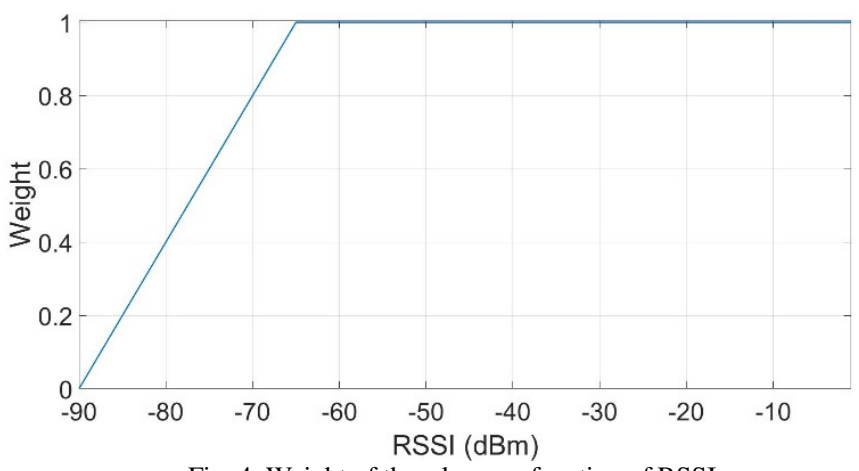

Fig. 4. Weight of the edge as a function of RSSI

The cost $C_{i}$ for the individual $i$ is then computed considering each edge weighted by its RSSI value according to (2) where $|E|$ is the number of elements included in set $E$.

$$
C_{i}=\sum_{e=1}^{|E|} w_{e}
$$

Then, for each of the 1000 individuals in the population a cost that corresponds to the cost function $C_{i}$ is assigned. The population is sorted by cost, and the 10 individuals with the lowest costs are used to contribute to the creation of the next generation of population $P_{\text {next }}$ by using a multi-point crossover method with two points and mutation. Specifically, a pair of individuals are randomly sampled from the 10 best individuals and their genomes are combined to obtain a new individual [15]. Then, the new individual is mutated by assigning for each AP a random channel from the two remaining options in the set of channels $\{1,6$, and 11$\}$ excluding the current channel, with a $1 \%$ probability. Afterwards, the new individual is included in the next generation of population $P_{\text {next. }}$

A set of 999 new individuals are created using this process and then, the best individual from the last generation of population is automatically included (this technique is called elitism). $P_{\text {next }}$ thus consists of 1000 individuals which are mutated combinations of the best individuals together with a direct copy from the previous generation. The computation of a new generation of population is stopped when there are 10 consecutive generations with no improvement in the lowest observed cost, or a maximum of 100 generations is reached. The best observed individual is chosen as the winner. The channel assignment of this individual is then implemented by the centralized management platform in the APs of the considered scenario. All the details on the multi-point crossover method can be found in [15].

\section{B. Wi-5 Algorithm}

This section presents the algorithm implemented in the centralized network management platform based on the Wi5 architecture and illustrated in Fig. 3. Specifically, the Wi-5 AP Channel Selection algorithm implemented in the controller selects the optimised channels in terms of interference impact for the different APs in the considered scenario.

Given $N$ APs and $F$ RF channels, we can quantify $I \in$ $\mathbb{R}^{N \times F}$, which is defined as the matrix of the interference impacts for $N$ APs and $F$ available channels. In this matrix, each $I_{i, f}$ element is the summation of the signals corresponding to channel $f$ when it is assigned to $A P_{i}$ and detected at other APs' locations.

The interference impact for $A P_{i}$ and its corresponding channel $f$ can be expressed as follows:

$$
\begin{aligned}
I_{i, f}= & \sum_{k \leq N, k \neq i} P_{i, k}(f)=\sum_{k \leq N, k \neq i} P_{i}^{t} \gamma_{i, k}(f) \theta_{i, k}(f)= \\
& P_{i}^{t} \sum_{k \leq N, k \neq i} \gamma_{i, k}(f) \theta_{i, k}(f)
\end{aligned}
$$

where $1 \leq f \leq F, l \leq i, k \leq N, P_{i, k}$ is the average power strength of the RF channel assigned to $A P_{i}$ and sensed at the close proximity of $A P_{k} . P_{i}^{t}$ is the transmission power level at $A P_{i}, \gamma_{i, k}$ is the channel gain between $A P_{i}$ and $A P_{k} . P_{i}^{t} \gamma_{i, k}(f)$ value corresponds to the RSSI scanned in the link connecting $A P_{i}$ to $A P_{k}$. Moreover, $\theta_{i, k}$ is the coefficient varying from 0 to 1 , representing the overlap between the channels assigned to $A P_{i}$ and $A P_{k}$. This coefficient will be zero for non-overlapping channels. An example of such overlap is provided in [16]. The matrix I reflects the interference impacts of APs' transmission powers in the objective function given below:

$$
A^{*}=\min _{A} \sum_{i \leq N} \sum_{\mathrm{f} \leq \mathrm{F}} G \times A^{T} . I
$$

where $G^{N \times N}$ is defined as the network topology matrix $G \in$ $\{0,1\}^{N \times N}$, where:

$$
g_{i j}=\left\{\begin{array}{c}
1, \quad \begin{array}{r}
\text { average power strength of } A P_{i} \text { around } A P_{j} \\
\text { exceeds a given threshold }
\end{array} \\
0, \quad \text { otherwise }
\end{array}\right.
$$

and $A^{F \times N}$ as the channel assignment matrix $A \in\{0,1\}^{F \times N}$, where: 


$$
a_{i j}=\left\{\begin{array}{lc}
1, & \text { if channel } i \text { is assigned to } A P_{j} \\
0, & \text { otherwise }
\end{array}\right.
$$

The resulting optimised channel assignment, $A^{*}$, aims to minimise the summation of these impacts throughout the network. More details about the binary Integer Linear Programing (ILP) approach we employed to solve (4) and the exact ILP coefficients can be found in [7]. As we have explained in Section II, in the Decision-Making module that provides the APs channel assignment, all the values needed for the computation of matrix I can be estimated and updated in real-time. However, for the study illustrated in this paper, the collected data introduced in Section II has been injected in the Decision-Making module and used for the computation of matrices $G, N$ and $I$ and then, for the execution of the optimised channel assignment $A^{*}$ in the considered scenario.

\section{PERFORMANCE RESUltS}

The channel assignments obtained through both algorithms were configured on the APs for the scenario illustrated in Fig. 1. Then, during one week, we collected a dataset on the radio channel conditions and traffic volumes every one minute in all the APs controlled by Domos. The resulting dataset has been averaged for all the APs during the week and used as the basis for comparing the algorithms. Specifically, the following parameters that allow to evaluate the quality of the data transmission have been considered during the data collection:

- Average Available Airtime: this is the percentage of time an AP is either transmitting, receiving or sensing that the frequency is idle, and averaged for all the Domos APs;

- Average Transmission Fail (Tx Fail): this is the number of times the AP transmits something but does not receive a successful ACK, (at the Wi-Fi MAC layer), which is computed every one minute during one week and averaged for all the Domos APs;

- Average Bad Transmission (Bad Tx): this is the percentage of time spent on transmitting packets which did not receive a successful ACK and averaged for all the Domos APs;

- Average Transmission Glitches (Glitch): this is the sum of the interruptions in the transmission or connectivity, and computed every one minute during one week, which can be mainly due to the interference level experienced in the APs. This value is then averaged for all the Domos APs.

\section{A. Validation of Data Collection}

In this subsection we validate the duration of the data collection. Specifically, in order to reach an accurate computation of the abovementioned performance metrics a sufficient number of samples was needed for each metric. To ensure that the averaged parameters collected every one minute during one week have properly converged to their real values, the $\gamma$ confidence interval tool has been used. Specifically, let us analyse the computation of Tx Fail as an example, indicated as $T$ for the sake of the simplicity from now on.
Let $\overline{T(N)}$ be defined as the sample mean of this parameter after we averaged a total of $N$ samples achieved through different collections. The $\gamma$ confidence interval is defined as the interval $\left[\overline{T_{\min }(N)}, \overline{T_{\max }(N)}\right.$ such that the real value of $T$ falls within such an interval with the following probability $\gamma$ :

$$
\operatorname{Pr}\left[T \in\left[\overline{T_{\min }(N)}, \overline{T_{\max }(N)}\right]\right]=\gamma
$$

If we take into account large-sample conditions, the values of the $\gamma$ confidence interval bounds $\overline{T_{\text {min }}(N)}$ and $\overline{T_{\max }(N)}$ after the average of $N$ collected samples are provided by:

$$
\begin{aligned}
& \overline{T_{\min }(N)}=\overline{T(N)}-z_{(1-\gamma) / 2} \frac{\overline{\sigma_{T}(N)}}{\sqrt{N}} \\
& \overline{T_{\max }(N)}=\overline{T(N)}+z_{(1-\gamma) / 2} \frac{\overline{\sigma_{T}(N)}}{\sqrt{N}}
\end{aligned}
$$

here, $Z_{(1-\gamma) / 2}=\phi^{-1}(1-(1-\gamma) / 2), \phi^{-1}(\cdot)$ represents the inverse of the normal cumulative distribution function and $\overline{\sigma_{T}(N)}$ denotes the standard deviation with $N$ collected samples. Note that any value $N>30$ is usually considered a large-sample condition [17]. Moreover, the greater the number of collected samples, the narrower the confidence interval, which means that the estimation obtained by the sample mean $\overline{T(N)}$ tends to converge to the real value. Therefore, the required number of collected samples $N$ which gives a sufficiently precise estimate of parameter $T$ by its sample mean $\overline{T(N)}$ is the first value that satisfies the following convergence condition:

$$
\overline{T_{\max }(N)}-\overline{T_{\min }(N)}<\eta \overline{T(N)}
$$

here $0<\eta<1$ is a parameter to be selected. Furthermore, this paper considers $\gamma=95 \%$, so that the term $z_{(1-\gamma) / 2}$ in (8) and (9) equals 1.96 [18]. Note that lower values of $\eta$ enable us to improve the asymptotic reliability at the expense of the time needed to get condition (10). Fig. 5 illustrates the evolution of the collected sample average $\overline{T(N)}$ and the $95 \%$ confidence interval bounds $\overline{T_{\max }(N)}$ and $\overline{T_{\min }(N)}$ as a function of the number of samples $N$.

From the figure it can be observed how a proper level of convergence in the computation of Tx Fail starts from $N=5000$ that corresponds to approximately $\eta=0.03$ in condition (10). Note also that the other performance metrics considered in the paper presented the same evolution and converged from around $N=5000$ for both algorithms. This result demonstrates that the collected data have converged to their real values after approximately half a week. Hence, we can claim that the results presented in the next subsection have converged to their realistic values.

\section{B. Comparison of the Algorithms}

Table I illustrates the performance results in terms of Available Airtime, Tx Fail, Bad Tx and Glitch for both the Domos and Wi-5 algorithms. From the table we can note that the Wi-5 algorithm provides better performance than the Domos approach. Specifically, the Wi-5 algorithm provides a slight improvement (i.e., a gain of 1.5\%) in terms of Available Airtime with respect to the Domos algorithm. The results in Table I also illustrate that the Wi-5 algorithm 
outperforms the Domos algorithm in terms of the Tx Fail and Bad Tx by $4 \%$. Finally, the Wi-5 algorithm outperforms the Domos algorithm by $12 \%$ in terms of Glitch.

From these results we can claim that the optimisation of the interference impact addressed by the Wi-5 algorithm provides the highest gain in terms of Glitch compared to the Domos algorithm that, in turn, optimize the cumulative interference of all neighbouring APs. This improvement in terms of interruptions in the transmission or connectivity illustrated through the study of the Glitch and achieved by the Wi-5 algorithm will undoubtedly have an effect also on the Quality of Experience (QoE) of the users that will be analysed as a part of our future works.

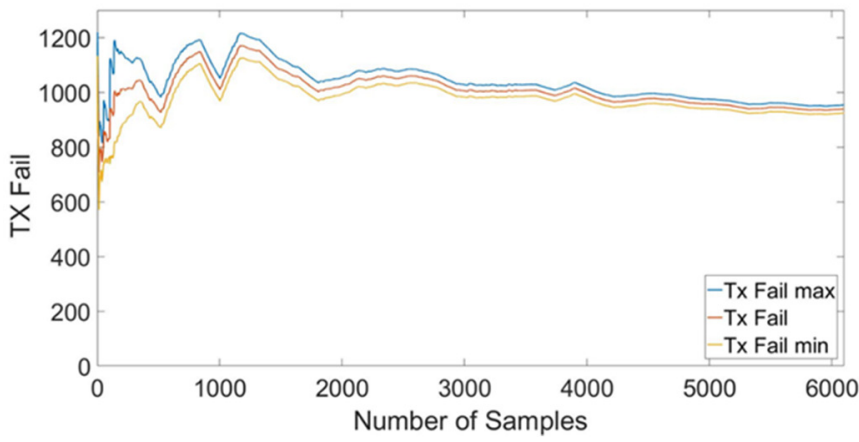

Fig. 5. Evolution of averaged Tx Fail value and 95\% confidence interval

TABLE I. PERFORMANCE RESUltS

\begin{tabular}{|l|l|l|l|l|}
\hline & Airtime (\%) & Tx Fail & Bad Tx (\%) & Glitch \\
\hline Domos & 66 & 974 & 0.80 & 1784 \\
\hline Wi-5 & 67 & 940 & 0.77 & 1561 \\
\hline Gain (\%) & $\mathbf{1 . 5}$ & $\mathbf{4}$ & $\mathbf{4}$ & $\mathbf{1 2}$ \\
\hline
\end{tabular}

\section{CONCLUSIONS AND FUTURE WORKS}

This paper has assessed the performance of optimised channel assignment algorithms for dense WLANs in a real world scenario represented by a housing complex located in Oslo, Norway. We have illustrated the implementation of the AP channel assignment algorithm designed in the context of the H2020 Wi-5 project which optimizes a novel metric called interference impact. The proposed AP channel selection algorithm is implemented in the Wi-5 architecture based on SDN, which manages the APs distributed in the WLAN of the considered scenario. A comparison has been performed against the current AP channel assignment algorithm implemented in the considered scenario by Domos, which optimize the cumulative interference experienced by the APs of the network. The performance analysis has revealed significant gains in terms quality of the data transmission obtained through the $\mathrm{Wi}-5 \mathrm{AP}$ channel selection algorithm, which will be leveraged by Domos.

As a part of our future works, we will investigate the benefits of the Wi-5 AP channel selection algorithm in terms of Quality of Experience (QoE) involving real Domos clients. Moreover, we will investigate further scenarios handled by the Domos centralized network management platform including 802.11n APs working also on the $5 \mathrm{GHz}$ band.

\section{ACKNOWLEDGMENT}

This work was funded in part by Domos AS and the research council of Norway under grant number 297244.

\section{REFERENCES}

[1] F. Bouhafs, M. Seyedebrahimi, A. Raschellà, M. Mackay, Q. Shi, "PerFlow Radio Resource Management to Mitigate Interference in Dense IEEE 802.11 Wireless LANs", IEEE Transaction on Mobile Computing (Early Access), Mar. 2019

[2] M. Achanta, "Method and Apparatus for Least Congested Channel Scan for Wireless Access Points," US Patent No. 20060072602, Apr. 2006.

[3] Qiao, et al. "Interference analysis and transmit power control in IEEE $802.11 \mathrm{a} / \mathrm{h}$ wireless LANs," IEEE/ACM Transaction on Networking (TON) vol. 15, issue 5, Oct.2007.

[4] K. Zhou, et al. "Channel Assignment for WLAN by Considering Overlapping Channels in SINR Interference Model", International Conference on Computing, Networking and Communications (ICNC), Maui, Hawaii, USA 30 Jan.- 02 Feb. 2012.

[5] A. Baid, et al. "Understanding channel selection dynamics in dense WiFi networks.” IEEE Com. Magazine, vol. 53, issue 1, Jan. 2015.

[6] E. Coronado, R. Riggio, J. Villalon, A. Garrido, "Wi-Balance: Channel-Aware User Association in Software-Defined Wi-Fi Networks", IEEE/IFIP Network Operations and Management Symposium (NOMS), Taipei, Taiwan, 23-27 Apr., 2018.K. Elissa, "Title of paper if known," unpublished.

[7] M. Seyedebrahimi, F. Bouhafs, A. Raschellà, M. Mackay, Q. Shi, "SDN-Based Channel Assignment Algorithm for Interference Management in Dense Wi-Fi Networks", European Conference on Networks and Communications (EuCNC), Athens, Greece, 27-30 Jun., 2016.

[8] M. Seyedebrahimi, F. Bouhafs, A. Raschellà, M. Mackay, Q. Shi, "Fine-Grained Radio Resource Management to Control Interference in Dense Wi-Fi Networks", IEEE Wireless Communications and Networking Conference (WCNC), San Francisco, CA, USA, 19-22 Mar., 2017.

[9] F. den Hartog, P. Kempker, B. Boltjes, A. Raschellà, F. Bouhafs, M. Seyedebrahimi, "A pathway to solving the Wi-Fi Tragedy of the Commons in apartment blocks", International Telecommunication Networks and Applications (ITNAC), Melbourne, Australia, 22-24, Nov., 2017.

[10] K. Sood, S. Liu, S. Yu, Y. Xiang, "Dynamic Access Point Association Using Software Defined Networking", International Telecommunication Networks and Applications Conference (ITNAC), Sydney, Australia,18-20 Nov. 2015.

[11] A. Raschellà, F. Bouhafs, M. Seyedebrahimi, M. Mackay, Q. Shi, "Quality of Service Oriented Access Point Selection Framework for Large Wi-Fi Networks", IEEE Transactions on Network and Service Management, Vol. 14, Issue 2, Pages 441-455, Jun. 2017.

[12] A. Raschellà, F. Bouhafs, M. Mackay, Q. Shi, J. Ortín, J.R. Gállego, M. Canales, "AP Selection Algorithm Based on a Potential Game for Large IEEE 802.11 WLANs", IEEE/IFIP Network Operations and Management Symposium (NOMS), Taipei, Taiwan, 23-27 Apr., 2018.

[13] F. Bouhafs, M. Mackay, A. Raschellà, Q. Shi, F. den Hartog, J. Saldana, J. Ruiz, J. Fernández-Navajas, R. Munilla, J. Almodovar, N. van Adrichem, "Wi-5: A Programming Architecture for Unlicensed Frequency Bands", IEEE Communications Magazine, Vol. 56, Issue 12, pp. 178 - 185, Dec. 2018.

[14] H2020 Wi-5 Project (What to do With the Wi-Fi Wild West), http://www.wi5.eu/.

[15] Stephen Marsland. Machine Learning: An Algorithmic Perspective, Second Edition. Chapman \& Hall/CRC, 2nd edition, 2014.

[16] A. Mishra, et al., "Weighted coloring based channel assignment for WLANs", ACM SIGMOBILE Mobile Computing and Communications Review Homepage vol. 9, issue 3, Jul. 2005.

[17] F. Bouali, O. Sallent, J. Pérez-Romero, R. Agustí, "Exploiting Knowledge Management for Supporting Multi-Band Spectrum Selection in Non-Stationary Environments", IEEE Transactions on Wireless Communications, vol.12, no 12, pp. 6228-6243, Jan. 2013.

[18] A. Raschellà, L. Militano, G. Araniti, A. Orsino, A. Iera, "Cognitive management strategies for dynamic spectrum access", Chapter in "Handbook of Cognitive Radio", Pages 1-35, Springer, May 2017. 